\title{
Hyers-Ulam Stability of Differentiation Operator on Hilbert Spaces of Entire Functions
}

\author{
Chun Wang ${ }^{1,2}$ and Tian-Zhou $\mathrm{Xu}^{1}$ \\ ${ }^{1}$ School of Mathematics and Statistics, Beijing Institute of Technology, Beijing 100081, China \\ ${ }^{2}$ Department of Mathematics, Changzhi University, Changzhi, Shanxi 046011, China \\ Correspondence should be addressed to Chun Wang; wangchun12001@163.com
}

Received 15 April 2014; Accepted 16 June 2014; Published 6 July 2014

Academic Editor: Zbigniew Leśniak

Copyright (c) 2014 C. Wang and T.-Z. Xu. This is an open access article distributed under the Creative Commons Attribution License, which permits unrestricted use, distribution, and reproduction in any medium, provided the original work is properly cited.

We investigate the Hyers-Ulam stability of differentiation operator on Hilbert spaces of entire functions. We give a necessary and sufficient condition in order that the operator has the Hyers-Ulam stability and also show that the best constant of Hyers-Ulam stability exists.

\section{Introduction}

In 1940, the first stability problem concerning group homomorphisms was raised by Ulam [1]. Let $G_{1}$ be a group and let $G_{2}$ be a metric group with a metric $d(\cdot, \cdot)$. Given any $\epsilon>0$, does there exist a $\delta>0$ such that if a function $h: G_{1} \rightarrow G_{2}$ satisfies the inequality $d(h(x y), h(x) h(y))<\delta$ for all $x, y \in$ $G_{1}$, then there exists a homomorphism $H: G_{1} \rightarrow G_{2}$ with $d(h(x), H(x))<\epsilon$ for all $x \in G_{1}$ ?

In the following years, Hyers affirmatively answered the question of Ulam for the case where $G_{1}$ and $G_{2}$ are Banach spaces (see [2]). Furthermore, the result of Hyers has been generalized by Rassias (see [3]).

Since then, the stability of many algebraic, differential, integral, operatorial, functional equations have been extensively investigated (see [4-17] and the references therein).

In this paper, we discuss the Hyers-Ulam stability of differentiation operator on Hilbert spaces of entire functions $E^{2}(\gamma)$ and give a necessary and sufficient condition in order that the operator has the Hyers-Ulam stability, and we show that the best constant of Hyers-Ulam stability exists.

\section{Hilbert Spaces of Entire Functions}

In this section, we describe the Hilbert spaces of entire functions in which the rest of our work is set and record their most basic properties. About the function spaces, we recommend the research papers $[18,19]$. For the sake of coherency we recall a few basic definitions, notions, and theorems from [18], and we also give some typical examples; in particular Fock space in these examples is a very important tool for quantum stochastic calculus in the case of quantum probability (see [20-22]).

Let us call an entire function $\gamma(z)=\sum_{n=0}^{\infty} \gamma_{n} z^{n}$ a comparison function if $\gamma_{n}>0$ for each $n$, and the sequence of ratios $\gamma_{n+1} / \gamma_{n}$ decreases to zero as $n$ increases to $\infty$. For each comparison function $\gamma(z)$, we define $E^{2}(\gamma)$ to be the Hilbert space of power series

$$
f(z)=\sum_{n=0}^{\infty} \widehat{f}(n) z^{n}
$$

for which

$$
\|f\|_{2, \gamma}^{2}:=\sum_{n=0}^{\infty} \gamma_{n}^{-2}|\widehat{f}(n)|^{2}<\infty
$$

It is easy to check that each element of $E^{2}(\gamma)$ is an entire function and that every sequence convergent in the norm of 
the space is uniformly convergent on compact subsets of the plane. In this case, the inner product of $E^{2}(\gamma)$ is given by

$$
\left\langle\sum_{n=0}^{\infty} a_{n} z^{n}, \sum_{n=0}^{\infty} b_{n} z^{n}\right\rangle:=\sum_{n=0}^{\infty} \gamma_{n}^{-2} a_{n} \overline{b_{n}}
$$

and the functions

$$
e_{n}(z)=\gamma_{n} z^{n} \quad(n=0,1,2, \ldots)
$$

form an orthonormal basis for $E^{2}(\gamma)$. We can see that the polynomials are dense in $E^{2}(\gamma)$.

Example 1. We consider the comparison function $\gamma_{1}(z)=$ $\sum_{k=0}^{\infty}(1 / \sqrt{k !}) z^{k}$; that is, $\gamma_{k}=1 / \sqrt{k !}$; by a simple calculation, we can see that $\left\|z^{k}\right\|=\sqrt{k !}$, and then $E^{2}\left(\gamma_{1}\right)$ is the famous Fock space.

Example 2. If we put comparison function $\gamma_{2}(z)=$ $\sum_{k=0}^{\infty}(1 / k !) z^{k}$, that is, $\gamma_{k}=1 / k$ !, we can see that $\left\|z^{k}\right\|=k$ ! on $E^{2}\left(\gamma_{2}\right)$.

Throughout this paper, let $D: E^{2}(\gamma) \rightarrow E^{2}(\gamma)$ be the differentiation operator defined by

$$
D(f)(z)=f^{\prime}(z), \quad\left(f \in E^{2}(\gamma), z \in \mathbb{C}\right) .
$$

An important result about $D$ is the following theorem.

Theorem 3 (see [18]). The operator $D$ is bounded on $E^{2}(\gamma)$ if and only if the sequence $\left\{w_{n}\right\}_{n=1}^{\infty}$ is bounded, where $w_{n}=$ $n \gamma_{n} / \gamma_{n-1}$.

By Theorem 3, we can obtain that the operator $D$ is unbounded on Fock space $E^{2}\left(\gamma_{1}\right)$, and it is bounded on $E^{2}\left(\gamma_{2}\right)$.

Throughout this paper, we suppose that the sequence $\left\{w_{n}\right\}_{n=1}^{\infty}$ is bounded.

\section{Hyers-Ulam Stability of Differentiation Operator}

Let $A, B$ be normed spaces and consider a mapping $T: A \rightarrow$ $B$. The following definition can be found in [14].

Definition 4. We say that $T$ has the Hyers-Ulam stability property (briefly, $T$ is $H U$-stable) if there exists a constant $K>0$ such that, for any $g \in T(A), \epsilon>0$, and $f \in A$ with $\|T f-g\| \leq \epsilon$, there exists an $f_{0} \in A$ with $T f_{0}=g$ and $\left\|f-f_{0}\right\| \leq K \epsilon$. The number $K$ is called a Hyers-Ulam stability constant (briefly HUS-constant) and the infimum of all HUS constants of $T$ is denoted by $K_{T}$; generally, $K_{T}$ is not a HUS constant of $T$ (see $[9,10])$.

Theorem 5. Let $D$ be the differentiation operator on the Hilbert spaces of entire functions $E^{2}(\gamma)$. Then the following statements are equivalent:

(a) D has Hyers-Ulam stability on $E^{2}(\gamma)$; (b) the sequence $\left\{1 / w_{n}\right\}_{n=1}^{\infty}$ is bounded, where $w_{n}=$ $n \gamma_{n} / \gamma_{n-1}$.

Proof. (b) $\Rightarrow$ (a). Suppose that the sequence $\left\{1 / w_{n}\right\}_{n=1}^{\infty}$ is bounded, and let $R=\sup \left\{1 / w_{n}: n \geq 1\right\}$. Since the polynomials are dense in $E^{2}(\gamma)$, we just need to show that $D$ has Hyers-Ulam stability on the polynomials dense subspace. For each $\epsilon \geq 0$, we take any two polynomials $f$ and $g$ that satisfy $\|D f-g\| \leq \epsilon, f$ and $g$ can be represented by the orthonormal basis. Then if $f(z)=\sum_{k=0}^{l}\left\langle f, e_{k}\right\rangle e_{k}, g(z)=$ $\sum_{k=0}^{m}\left\langle g, e_{k}\right\rangle e_{k}$, we have

$$
\begin{aligned}
D f(z) & =\sum_{k=1}^{l} k\left\langle f, e_{k}\right\rangle \gamma_{k} z^{k-1} \\
& =\sum_{k=1}^{l} \frac{k \gamma_{k}}{\gamma_{k-1}}\left\langle f, e_{k}\right\rangle \gamma_{k-1} z^{k-1} \\
& =\sum_{k=1}^{l} \frac{k \gamma_{k}}{\gamma_{k-1}}\left\langle f, e_{k}\right\rangle e_{k-1} \\
& =\sum_{k=0}^{l-1} \frac{(k+1) \gamma_{k+1}}{\gamma_{k}}\left\langle f, e_{k+1}\right\rangle e_{k} \\
& =\sum_{k=0}^{l-1} w_{k+1}\left\langle f, e_{k+1}\right\rangle e_{k} .
\end{aligned}
$$

For any nonnegative integers $m$ and $l$ such that $m \geq l$, we can get

$$
\begin{aligned}
\|D f-g\|^{2} & =\left\|\sum_{k=0}^{l-1} w_{k+1}\left\langle f, e_{k+1}\right\rangle e_{k}-\sum_{k=0}^{m}\left\langle g, e_{k}\right\rangle e_{k}\right\|^{2} \\
& =\| \sum_{k=0}^{l-1} w_{k+1}\left\langle f, e_{k+1}\right\rangle e_{k}-\sum_{k=0}^{l-1}\left\langle g, e_{k}\right\rangle e_{k} \\
& =\| \sum_{k=0}^{l-1}\left[w_{k+1}\left\langle f, e_{k+1}\right\rangle-\left\langle g, e_{k}\right\rangle\right] e_{k} \\
& +\sum_{k=1}^{m}\left\langle g, e_{k}\right\rangle e_{k}\left\|^{2}\left|\sum_{k=l}^{m}\left[-\left\langle g, e_{k}\right\rangle\right] e_{k} \|^{2}\left\langle f, e_{k+1}\right\rangle-\left\langle g, e_{k}\right\rangle\right|^{2}+\sum_{k=l}^{m}\left|\left\langle g, e_{k}\right\rangle\right|^{2}\right.
\end{aligned}
$$

Hence,

$$
\sum_{k=0}^{l-1}\left|w_{k+1}\left\langle f, e_{k+1}\right\rangle-\left\langle g, e_{k}\right\rangle\right|^{2}+\sum_{k=l}^{m}\left|\left\langle g, e_{k}\right\rangle\right|^{2} \leq \epsilon^{2} .
$$


Let $f_{0} \in E^{2}(\gamma)$ be the function defined by

$$
f_{0}(z)=\left\langle f, e_{0}\right\rangle e_{0}+\sum_{k=1}^{m+1} \frac{\left\langle g, e_{k-1}\right\rangle \gamma_{k-1}}{k \gamma_{k}} e_{k} .
$$

It is easy to check that $D f_{0}=g$, also; from (8), we obtain

$$
\begin{aligned}
& \left\|f-f_{0}\right\|^{2}=\| \sum_{k=0}^{l}\left\langle f, e_{k}\right\rangle e_{k} \\
& -\left(\left\langle f, e_{0}\right\rangle e_{0}+\sum_{k=1}^{m+1} \frac{\left\langle g, e_{k-1}\right\rangle \gamma_{k-1}}{k \gamma_{k}} e_{k}\right) \|^{2} \\
& =\| \sum_{k=1}^{l}\left[\left\langle f, e_{k}\right\rangle-\frac{\left\langle g, e_{k-1}\right\rangle \gamma_{k-1}}{k \gamma_{k}}\right] e_{k} \\
& +\sum_{k=l+1}^{m+1} \frac{\left\langle g, e_{k-1}\right\rangle \gamma_{k-1}}{k \gamma_{k}} e_{k} \|^{2} \\
& =\sum_{k=1}^{l}\left|\left\langle f, e_{k}\right\rangle-\frac{\left\langle g, e_{k-1}\right\rangle \gamma_{k-1}}{k \gamma_{k}}\right|^{2} \\
& +\sum_{k=l+1}^{m+1}\left|\frac{\left\langle g, e_{k-1}\right\rangle \gamma_{k-1}}{k \gamma_{k}}\right|^{2} \\
& =\sum_{k=1}^{l}\left|\left\langle f, e_{k}\right\rangle-\left\langle g, e_{k-1}\right\rangle \cdot \frac{1}{w_{k}}\right|^{2} \\
& +\sum_{k=l+1}^{m+1}\left|\left\langle g, e_{k-1}\right\rangle \cdot \frac{1}{w_{k}}\right|^{2} \\
& =\sum_{k=1}^{l}\left|w_{k}\left\langle f, e_{k}\right\rangle-\left\langle g, e_{k-1}\right\rangle\right|^{2}\left|\frac{1}{w_{k}}\right|^{2} \\
& +\sum_{k=l+1}^{m+1}\left|\left\langle g, e_{k-1}\right\rangle\right|^{2}\left|\frac{1}{w_{k}}\right|^{2} \\
& \leq\left\{\sum_{k=1}^{l}\left|w_{k}\left\langle f, e_{k}\right\rangle-\left\langle g, e_{k-1}\right\rangle\right|^{2}\right. \\
& \left.+\sum_{k=l+1}^{m+1}\left|\left\langle g, e_{k-1}\right\rangle\right|^{2}\right\} \cdot R^{2} \\
& \leq R^{2} \epsilon^{2} .
\end{aligned}
$$

Next, assume that $m=l-1$. It follows that

$$
\begin{aligned}
\|D f-g\|^{2} & =\left\|\sum_{k=0}^{l-1} w_{k+1}\left\langle f, e_{k+1}\right\rangle e_{k}-\sum_{k=0}^{l-1}\left\langle g, e_{k}\right\rangle e_{k}\right\|^{2} \\
& =\left\|\sum_{k=0}^{l-1}\left[w_{k+1}\left\langle f, e_{k+1}\right\rangle-\left\langle g, e_{k}\right\rangle\right] e_{k}\right\|^{2} \\
& =\sum_{k=0}^{l-1}\left|w_{k+1}\left\langle f, e_{k+1}\right\rangle-\left\langle g, e_{k}\right\rangle\right|^{2} .
\end{aligned}
$$

Hence,

$$
\sum_{k=0}^{l-1}\left|w_{k+1}\left\langle f, e_{k+1}\right\rangle-\left\langle g, e_{k}\right\rangle\right|^{2} \leq \epsilon^{2} .
$$

Let

$$
f_{0}(z)=\left\langle f, e_{0}\right\rangle e_{0}+\sum_{k=1}^{l} \frac{\left\langle g, e_{k-1}\right\rangle \gamma_{k-1}}{k \gamma_{k}} e_{k} .
$$

Then $D f_{0}=g$; by (12), we have

$$
\begin{aligned}
\left\|f-f_{0}\right\|^{2}= & \| \sum_{k=0}^{l}\left\langle f, e_{k}\right\rangle e_{k} \\
& -\left(\left\langle f, e_{0}\right\rangle e_{0}+\sum_{k=1}^{l} \frac{\left\langle g, e_{k-1}\right\rangle \gamma_{k-1}}{k \gamma_{k}} e_{k}\right) \|^{2} \\
= & \left\|\sum_{k=1}^{l}\left[\left\langle f, e_{k}\right\rangle-\frac{\left\langle g, e_{k-1}\right\rangle \gamma_{k-1}}{k \gamma_{k}}\right] e_{k}\right\|^{2} \\
= & \sum_{k=1}^{l}\left|\left\langle f, e_{k}\right\rangle-\frac{\left\langle g, e_{k-1}\right\rangle \gamma_{k-1}}{k \gamma_{k}}\right|^{2} \\
= & \sum_{k=0}^{l-1}\left|\left\langle f, e_{k+1}\right\rangle-\frac{\left\langle g, e_{k}\right\rangle \gamma_{k}}{(k+1) \gamma_{k+1}}\right|^{2} \\
= & \sum_{k=0}^{l-1}\left|\left\langle f, e_{k+1}\right\rangle-\left\langle g, e_{k}\right\rangle \frac{1}{w_{k+1}}\right|^{2} \\
= & \sum_{k=0}^{l-1}\left|w_{k+1}\left\langle f, e_{k+1}\right\rangle-\left\langle g, e_{k}\right\rangle\right|^{2}\left|\frac{1}{w_{k+1}}\right|^{2} \\
\leq & R^{2} \epsilon^{2} .
\end{aligned}
$$


Finally, if $m<l-1$, we get

$$
\begin{aligned}
\|D f-g\|^{2}= & \left\|\sum_{k=0}^{l-1} w_{k+1}\left\langle f, e_{k+1}\right\rangle e_{k}-\sum_{k=0}^{m}\left\langle g, e_{k}\right\rangle e_{k}\right\|^{2} \\
= & \| \sum_{k=0}^{m} w_{k+1}\left\langle f, e_{k+1}\right\rangle e_{k} \\
& +\sum_{k=m+1}^{l-1} w_{k+1}\left\langle f, e_{k+1}\right\rangle e_{k}-\sum_{k=0}^{m}\left\langle g, e_{k}\right\rangle e_{k} \|^{2} \\
= & \| \sum_{k=0}^{m}\left[w_{k+1}\left\langle f, e_{k+1}\right\rangle-\left\langle g, e_{k}\right\rangle\right] e_{k} \\
& +\sum_{k=0}^{m}\left|\sum_{k=m+1}^{l-1}\left\langle f, e_{k+1}\right\rangle-\left\langle g, e_{k}\right\rangle\right|^{2}\left\langle f, e_{k+1}\right\rangle e_{k} \|^{2} \\
& +\sum_{k=m+1}^{l-1}\left|w_{k+1}\left\langle f, e_{k+1}\right\rangle\right|^{2} .
\end{aligned}
$$

Hence,

$$
\sum_{k=0}^{m}\left|w_{k+1}\left\langle f, e_{k+1}\right\rangle-\left\langle g, e_{k}\right\rangle\right|^{2}+\sum_{k=m+1}^{l-1}\left|w_{k+1}\left\langle f, e_{k+1}\right\rangle\right|^{2} \leq \epsilon^{2} .
$$

Thus, from (16), it follows that

$$
\begin{aligned}
\left\|f-f_{0}\right\|^{2}= & \| \sum_{k=0}^{l}\left\langle f, e_{k}\right\rangle e_{k} \\
& -\left(\left\langle f, e_{0}\right\rangle e_{0}+\sum_{k=1}^{m+1} \frac{\left\langle g, e_{k-1}\right\rangle \gamma_{k-1}}{k \gamma_{k}} e_{k}\right) \|^{2} \\
= & \| \sum_{k=1}^{m+1}\left\langle f, e_{k}\right\rangle e_{k}+\sum_{k=m+2}^{l}\left\langle f, e_{k}\right\rangle e_{k} \\
& -\sum_{k=1}^{m+1} \frac{\left\langle g, e_{k-1}\right\rangle \gamma_{k-1}}{k \gamma_{k}} e_{k}^{2} \|^{m+1}\left[\left\langle f, e_{k}\right\rangle-\frac{\left\langle g, e_{k-1}\right\rangle \gamma_{k-1}}{k \gamma_{k}}\right] e_{k} \\
& +\sum_{k=1}^{l}\left\langle f, e_{k}\right\rangle e_{k} \|^{2}
\end{aligned}
$$

$$
\begin{aligned}
= & \sum_{k=1}^{m+1}\left|\left\langle f, e_{k}\right\rangle-\frac{\left\langle g, e_{k-1}\right\rangle \gamma_{k-1}}{k \gamma_{k}}\right|^{2} \\
& +\sum_{k=m+2}^{l}\left|\left\langle f, e_{k}\right\rangle\right|^{2} \\
= & \sum_{k=0}^{m}\left|\left\langle f, e_{k+1}\right\rangle-\frac{\left\langle g, e_{k}\right\rangle \gamma_{k}}{(k+1) \gamma_{k+1}}\right|^{2} \\
& +\sum_{k=m+1}^{l-1}\left|\left\langle f, e_{k+1}\right\rangle\right|^{2} \\
= & \sum_{k=0}^{m}\left|\left\langle f, e_{k+1}\right\rangle-\left\langle g, e_{k}\right\rangle \frac{1}{w_{k+1}}\right|^{2} \\
& +\sum_{k=m+1}^{l-1}\left|w_{k+1}\left\langle f, e_{k+1}\right\rangle\right|^{2} \cdot\left|\frac{1}{w_{k+1}}\right|^{2} \\
& +\sum_{k=m+1}^{l-1}\left|\left\langle f, e_{k+1}\right\rangle\right|^{2} \\
= & \sum_{k=0}^{m}\left|w_{k+1}\left\langle f, e_{k+1}\right\rangle-\left\langle g, e_{k}\right\rangle\right|^{2} \cdot\left|\frac{1}{w_{k+1}}\right|^{2}
\end{aligned}
$$$$
\leq\left\{\sum_{k=0}^{m}\left|w_{k+1}\left\langle f, e_{k+1}\right\rangle-\left\langle g, e_{k}\right\rangle\right|^{2}\right.
$$$$
\left.+\sum_{k=m+1}^{l-1}\left|w_{k+1}\left\langle f, e_{k+1}\right\rangle\right|^{2}\right\} \cdot R^{2}
$$$$
\leq R^{2} \epsilon^{2} \text {. }
$$

Therefore, (a) holds.

$(\mathrm{a}) \Rightarrow(\mathrm{b})$. Suppose that $D$ is stable with Hyers-Ulam stability constant $K$. For any nonnegative integer $k$, let $f(z)=$ $\left(1 / w_{k+1}\right) e_{k+1}$. Then $\|D f(z)\|=1$, so there exists $f_{0}$ such that $D f_{0}=0$ and $\left\|f(z)-f_{0}(z)\right\| \leq K$. Hence,

$$
\frac{1}{w_{k+1}}-\left\|f_{0}(z)\right\|=\|f(z)\|-\left\|f_{0}(z)\right\| \leq\left\|f(z)-f_{0}(z)\right\| \leq K,
$$

and consequently $0<1 / w_{k+1} \leq K+\left\|f_{0}(z)\right\|$ for any nonnegative integer $k$. This completes the proof.

Example 6. We consider the comparison function $\gamma_{2}(z)=$ $\sum_{k=0}^{\infty}(1 / k !) z^{k}$; that is, $\gamma_{k}=1 / k$ !; by a simple calculation, we have $w_{k}=1$ and $1 / w_{k}=1(k=1,2, \ldots)$, and hence operator $D$ has Hyers-Ulam stability on the $E^{2}\left(\gamma_{2}\right)$.

Example 7. If we put comparison function $\gamma_{3}(z)=$ $\sum_{k=0}^{\infty}\left(1 /(k !)^{2}\right) z^{k}$, that is, $\gamma_{k}=1 /(k !)^{2}$, we have $w_{k}=1 / k$ and 
$1 / w_{k}=k(k=1,2, \ldots),\left\{1 / w_{k}\right\}_{k=1}^{\infty}$ is unbounded, and hence operator $D$ is not Hyers-Ulam stable on the $E^{2}\left(\gamma_{3}\right)$.

Example 8. We consider the comparison function $\gamma_{4}(z)=1+$ $2 z+\sum_{k=2}^{\infty}(1 / k \cdot k !) z^{k}$; we get $\gamma_{0}=1, \gamma_{1}=2, \gamma_{k}=1 / k \cdot k !$ $(k=2,3, \ldots)$, and by a simple calculation, we have $w_{1}=2$, $w_{2}=1 / 4, w_{k}=(k-1) / k(k=3,4, \ldots)$ and $1 / w_{1}=1 / 2$, $1 / w_{2}=4,1 / w_{k}=1+(1 /(k-1))(k=3,4, \ldots)$, where $\left\{1 / w_{k}\right\}_{k=1}^{\infty}$ is bounded; hence, operator $D$ has Hyers-Ulam stability on the $E^{2}\left(\gamma_{4}\right)$.

Remark 9. From Theorem 5 and Examples 6-8, we can see that the Hyers-Ulam stability of differentiation operator $D$ on Hilbert spaces of entire functions $E^{2}(\gamma)$ depends on the comparison functions $\gamma(z)$. It shows that the comparison functions affects the behaviors of the operators and the functions in the corresponding Hilbert space $E^{2}(\gamma)$.

Next, we will show that the best constant of Hyers-Ulam stability exists.

Theorem 10. If differentiation operator D has the Hyers-Ulam stability on Hilbert spaces of entire functions $E^{2}(\gamma)$, then $K_{D}=$ $\sup \left\{1 / w_{n}: n \geq 1\right\}$ and $K_{D}$ is a HUS constant of $D$.

Proof. Suppose that $D$ has Hyers-Ulam stability on $E^{2}(\gamma)$. By the proof of Theorem 5, we know that $\sup \left\{1 / w_{n}: n \geq 1\right\}$ is a constant of the Hyers-Ulam stability of differentiation operator $D$. Next, we show that it is the infimum of all the Hyers-Ulam stability constants. Let $K<\infty$ be an arbitrary Hyers-Ulam stability constant for $D$; put $f(z)=$ $\left(1 / w_{k+1}\right) e_{k+1}$, and for any nonnegative integer $k$, we can obtain $\|D f(z)\|=1$, so there exists $f_{0}=c_{0}$, such that $D f_{0}=0$ and $\left\|f(z)-c_{0}\right\| \leq K$, and hence

$$
\frac{1}{w_{k+1}} \leq\left\|\frac{1}{w_{k+1}} e_{k+1}-c_{0}\right\|=\left\|f(z)-c_{0}\right\| \leq K,
$$

and so $\sup \left\{1 / w_{n}: n \geq 1\right\} \leq K$.

\section{Conflict of Interests}

The authors declare that there is no conflict of interests regarding the publication of this paper.

\section{Acknowledgments}

The authors are very grateful to the editor and reviewers for their valuable comments and suggestions on the paper. This work is supported by the National Natural Science Foundation of China (Grant no. 11171022).

\section{References}

[1] S. M. Ulam, A Collection of Mathematical Problems, Interscience, New York, NY, USA, 1960.

[2] D. H. Hyers, "On the stability of the linear functional equation," Proceedings of the National Academy of Sciences of the United States of America, vol. 27, pp. 222-224, 1941.
[3] Th. M. Rassias, "On the stability of the linear mapping in Banach spaces," Proceedings of the American Mathematical Society, vol. 72, no. 2, pp. 297-300, 1978.

[4] J. Brzdęk and S.-M. Jung, "A note on stability of an operator linear equation of the second order," Abstract and Applied Analysis, vol. 2011, Article ID 602713, 15 pages, 2011.

[5] J. Brzdek, D. Popa, and B. Xu, "On approximate solutions of the linear functional equation of higher order," Journal of Mathematical Analysis and Applications, vol. 373, no. 2, pp. 680689, 2011

[6] K. Ciepliński, "Generalized stability of multi-additive mappings," Applied Mathematics Letters, vol. 23, no. 10, pp. 12911294, 2010.

[7] K. Ciepliński, "Applications of fixed point theorems to the Hyers-Ulam stability of functional equations-a survey," Annals of Functional Analysis, vol. 3, no. 1, pp. 151-164, 2012.

[8] P. Găvruţa, "A generalization of the Hyers-Ulam-Rassias stability of approximately additive mappings," Journal of Mathematical Analysis and Applications, vol. 184, no. 3, pp. 431-436, 1994.

[9] O. Hatori, K. Kobayashi, T. Miura, H. Takagi, and S. E. Takahasi, "On the best constant of Hyers-Ulam stability," Journal of Nonlinear and Convex Analysis, vol. 5, no. 3, pp. 387-393, 2004.

[10] G. Hirasawa and T. Miura, "Hyers-Ulam stability of a closed operator in a Hilbert space," Bulletin of the Korean Mathematical Society, vol. 43, no. 1, pp. 107-117, 2006.

[11] T. Miura, G. Hirasawa, and S. E. Takahasi, "Ger-type and HyersUlam stabilities for the first-order linear differential operators of entire functions," International Journal of Mathematics and Mathematical Sciences, no. 22, pp. 1151-1158, 2004.

[12] D. Popa and I. Raşa, "The Fréchet functional equation with application to the stability of certain operators," Journal of Approximation Theory, vol. 164, no. 1, pp. 138-144, 2012.

[13] D. Popa and I. Raşa, “On the stability of some classical operators from approximation theory," Expositiones Mathematicae, vol. 31, no. 3, pp. 205-214, 2013.

[14] H. Takagi, T. Miura, and S. E. Takahasi, "Essential norms and stability constants of weighted composition operators on $\mathrm{C}(\mathrm{X})$," Bulletin of the Korean Mathematical Society, vol. 40, no. 4, pp. 583-591, 2003.

[15] T. Z. Xu, "Approximate multi-Jensen, multi-Euler-Lagrange additive and quadratic mappings in $n$-Banach spaces," Abstract and Applied Analysis, vol. 2013, Article ID 648709, 12 pages, 2013.

[16] T. Z. Xu, "On the stability of multi-Jensen mappings in $\beta$ normed spaces," Applied Mathematics Letters, vol. 25, no. 11, pp. 1866-1870, 2012.

[17] T. Z. Xu, Z. Yang, and J. M. Rassias, "Direct and fixed point approaches to the stability of an AQ-functional equation in non-Archimedean normed spaces," Journal of Computational Analysis and Applications, vol. 17, no. 4, pp. 697-706, 2014.

[18] K. C. Chan and J. H. Shapiro, "The cyclic behavior of translation operators on Hilbert spaces of entire functions," Indiana University Mathematics Journal, vol. 40, no. 4, pp. 1421-1449, 1991.

[19] G. A. Chacón, G. R. Chacón, and J. Giménez, "Composition operators on spaces of entire functions," Proceedings of the American Mathematical Society, vol. 135, no. 7, pp. 2205-2218, 2007. 
[20] V. Crismale, "Quantum stochastic calculus on interacting Fock spaces: semimartingale estimates and stochastic integral," Communications on Stochastic Analysis, vol. 1, no. 2, pp. 321-341, 2007.

[21] B. K. Das, J. M. Lindsay, and O. Tripak, "Sesquilinear quantum stochastic analysis in Banach space," Journal of Mathematical Analysis and Applications, vol. 409, no. 2, pp. 1032-1051, 2014.

[22] U. C. Ji and N. Obata, "Quantum stochastic integral representations of Fock space operators," Stochastics, vol. 81, no. 3-4, pp. 367-384, 2009. 


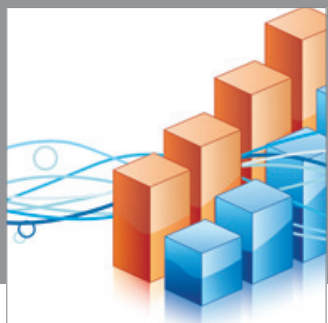

Advances in

Operations Research

mansans

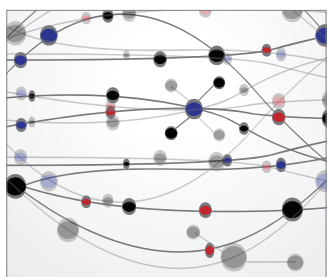

The Scientific World Journal
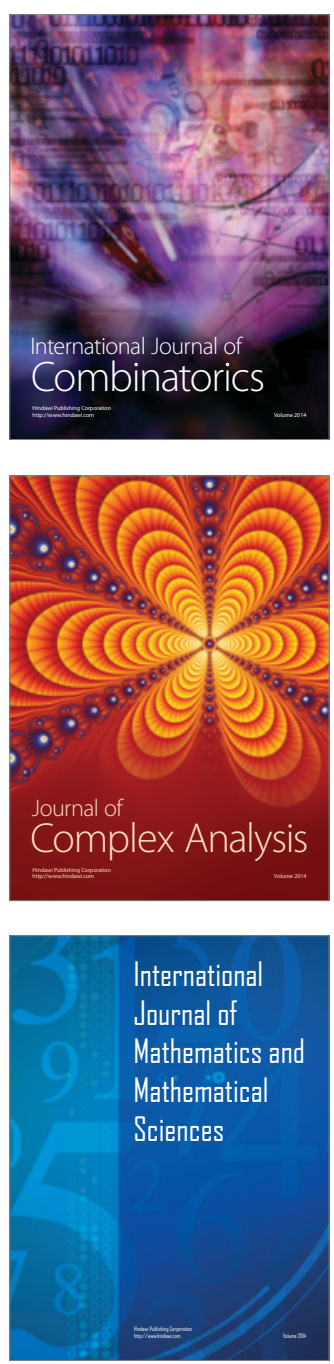
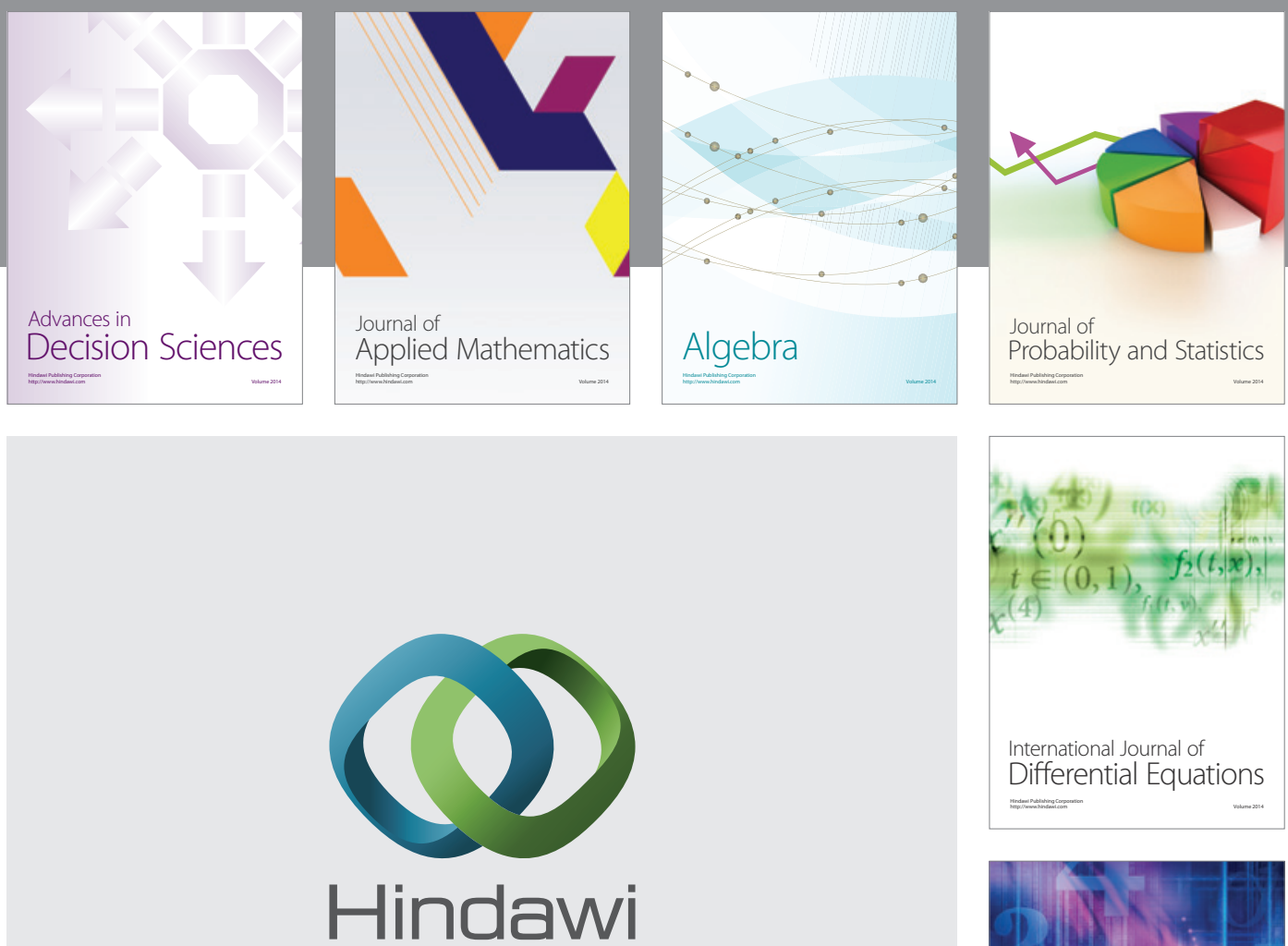

Submit your manuscripts at http://www.hindawi.com
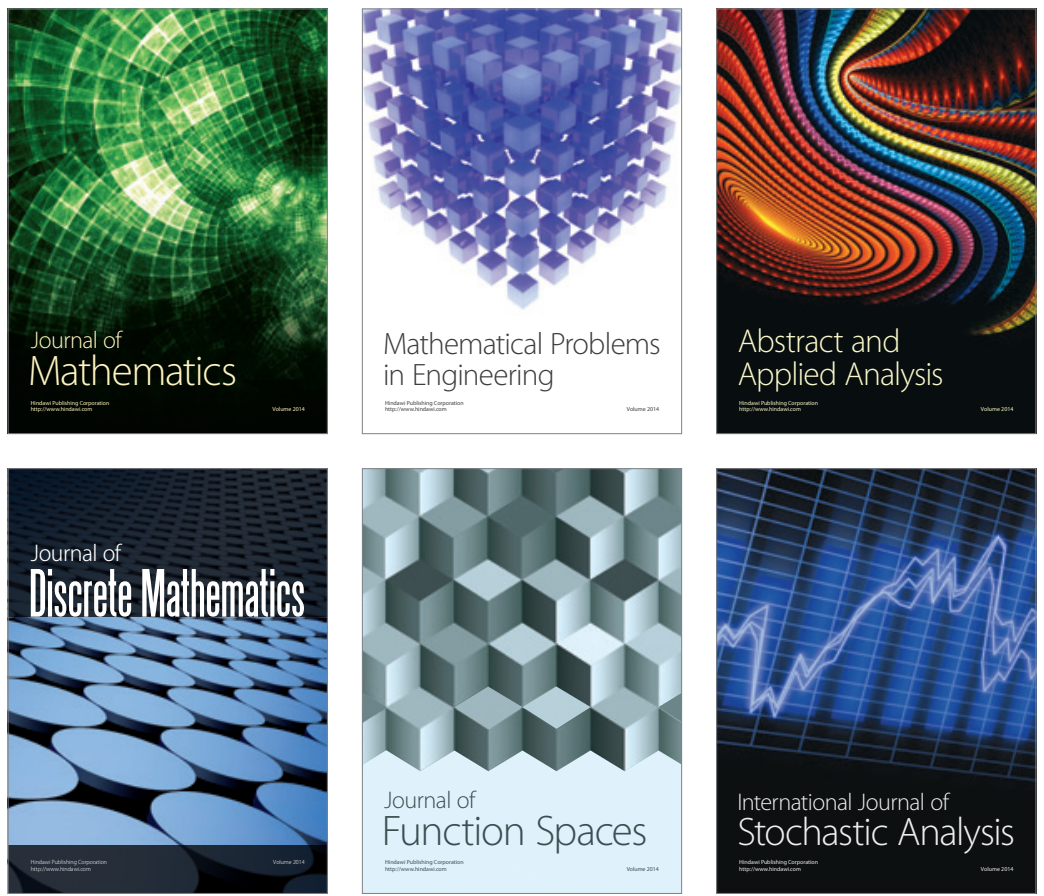

Journal of

Function Spaces

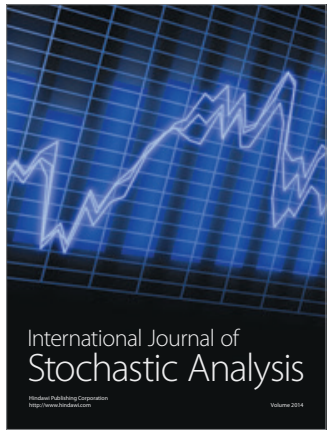

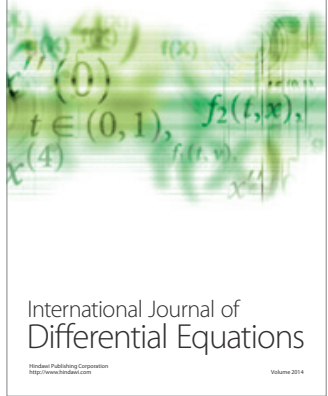
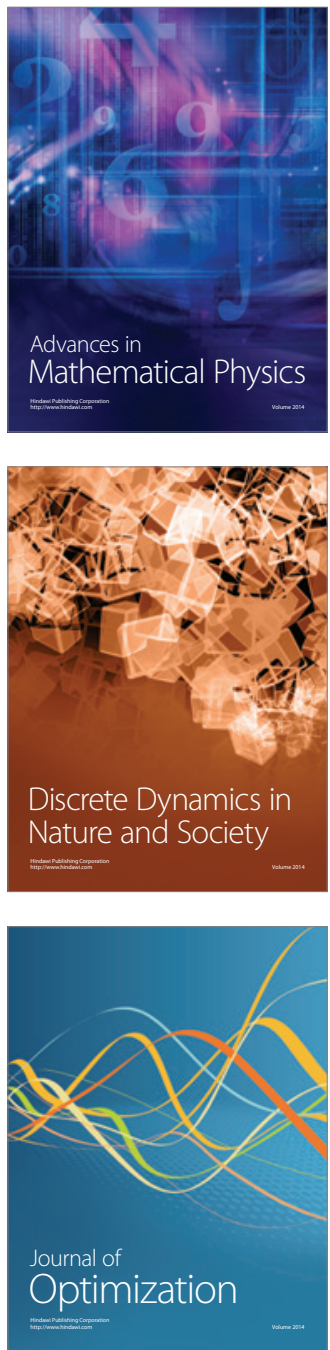